A renormalized potential-following propagation algorithm for solving the coupledchannels equations

Tijs Karman, Liesbeth M. C. Janssen, Rik Sprenkels, and Gerrit C. Groenenboom'

Citation: The Journal of Chemical Physics 141, 064102 (2014); doi: 10.1063/1.4891809

View online: http://dx.doi.org/10.1063/1.4891809

View Table of Contents: http://aip.scitation.org/toc/jcp/141/6

Published by the American Institute of Physics

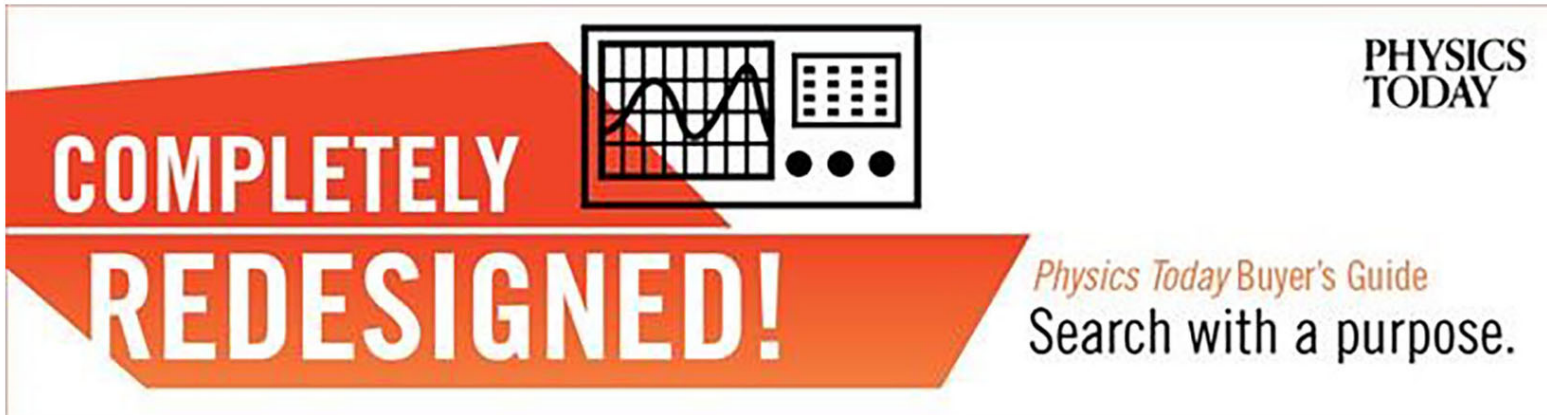




\title{
A renormalized potential-following propagation algorithm for solving the coupled-channels equations
}

\author{
Tijs Karman, ${ }^{1}$ Liesbeth M. C. Janssen, ${ }^{2}$ Rik Sprenkels, ${ }^{1}$ and Gerrit C. Groenenboom ${ }^{1, a)}$ \\ ${ }^{1}$ Theoretical Chemistry, Institute for Molecules and Materials, Radboud University Nijmegen, Nijmegen, \\ The Netherlands \\ ${ }^{2}$ Department of Chemistry, Columbia University, 3000 Broadway, New York, New York 10027, USA
}

(Received 16 May 2014; accepted 21 July 2014; published online 8 August 2014)

\begin{abstract}
We derive a general renormalized potential-following propagation method that efficiently solves the coupled-channels equations. The step size is variable, the method is compatible with reactive boundary conditions, and the algorithm may be combined with other renormalized algorithms, such as renormalized Numerov. We diagonalize the coupling matrix and consider piece-wise constant and linear reference potentials. The constant reference potential algorithm is very simple to implement, yet for multichannel problems almost as accurate as the linear reference potential method. The applicability of the proposed algorithms to realistic problems is demonstrated for cold collisions of $\mathrm{NH}$ radicals. The renormalized approach has the advantage of producing wave functions in a straightforward way, which is illustrated for a shape resonance in $\mathrm{NH}-\mathrm{NH}$ collisions. These scattering wave functions can be used to study ultracold photoassociation and near-threshold photodissociation. (C) 2014 AIP Publishing LLC. [http://dx.doi.org/10.1063/1.4891809]
\end{abstract}

\section{INTRODUCTION}

Molecular scattering is described time-independently by the coupled-channels equations, i.e., by a set of coupled second-order differential equations in the intermolecular distance, $R$, where the first derivative is absent. To ensure numerical stability, one typically propagates derived quantities, rather than propagating the solutions of the equations directly. ${ }^{1,2}$ The most widely used formulations are logderivative and renormalized propagation. ${ }^{3,4}$ In log-derivative algorithms, one propagates $Y$, the ratio of the wave function and its first derivative, $\Psi^{\prime}=Y \Psi$. In renormalized methods, one propagates $Q$, the ratio of the solution in two points, defined by $\Psi_{i-1}=Q_{i} \Psi_{i}$, where the subscript refers to the radial grid point. Both approaches resolve the instability. However, neither approach is computationally superior to the other. The renormalized method has the advantage that it is very straightforward to reconstruct the complete wave function, once it is known in one point, simply by repeated multiplication with the calculated Q-matrices. Also, the Q-matrices can be used to propagate integrals over the wave function along with the wave function itself, which allows one to calculate matrix elements without explicit reconstruction of many-component wave functions. ${ }^{5}$

The accuracy of a method is determined by the level of approximation that is used to derive recurrence relations. It is convenient to distinguish between two types of approximations, namely, solution-following and potential-following propagators. ${ }^{6}$ As the name suggests, solution-following propagators approximate the wave function, typically by using a truncated Taylor expansion of the solution. Potentialfollowing algorithms approximate the potential by a simple

\footnotetext{
a)Electronic mail: gerritg@ theochem.ru.nl
}

form, and propagate in terms of the exact solutions to the coupled-channels equations for the approximated potential. Typically, solution-following methods are efficient around the minimum of the potential, where the potential varies rapidly. ${ }^{7}$ Asymptotically, however, the propagation step size is limited by the finite de Broglie wavelength of the solution. Potentialfollowing methods become more efficient and asymptotically exact, as the potential becomes constant in the long range. ${ }^{8}$

The original renormalized Numerov algorithm is a solution-following method that uses an equidistant grid in the radial coordinate, $R$, and a Taylor expansion of the solution up to fifth order in the grid spacing. ${ }^{1,2}$ This method has been improved upon by Thorlacius and Cooper ${ }^{9}$ by summing the Taylor series to infinite order, with approximations that are exact in case of a constant potential. Colavecchia et al. ${ }^{10}$ have combined the enhanced renormalized Numerov method with a smooth variable discretization, that allows one to use an adiabatic basis without including derivative couplings. A generalization of the renormalized Numerov method to nonequidistant radial grids has been presented by Vigo-Aguiar and Ramos. ${ }^{11}$ The original log-derivative propagator is also a solution-following algorithm. ${ }^{1,2}$ For log-derivative propagation, more efficient solution-following propagators exist, such as constant reference potential type improvements, ${ }^{12}$ and the symplectic log-derivative propagators. ${ }^{13}$ There also exist potential-following log-derivative algorithms that approximate the potential by constant or linear segments. ${ }^{8,14-16}$ However, a renormalized potential-following propagator does not yet exist.

Potential-following algorithms are computationally very efficient for scattering calculations where one has to propagate to large distances. This is the case, e.g., for collisions of polar molecules, with strong long-ranged dipole-dipole interactions, ${ }^{17}$ especially in the case of cold and ultracold 
collisions. ${ }^{18,19}$ For such calculations, one could use existing log-derivative propagators. ${ }^{8}$ However, a renormalized method has the advantage that it is straightforward to calculate scattering wave functions. This is useful for the analysis of scattering resonances in cold collisions. ${ }^{20,21}$ Scattering wave functions for ultracold collisions are also required to describe nearthreshold photodissociation, ${ }^{22,23}$ and photoassociation of ultracold atoms. ${ }^{24}$

In this work, we present a general method for renormalized potential-following propagation. The step size is variable, and the method may be combined with solutionfollowing algorithms in the short range. We explicitly consider piece-wise constant and linear approximations to the potential, which yield renormalized analogues of existing logderivative propagators. Implementations of these algorithms are made available in the supplementary material as Scilab and Matlab scripts. ${ }^{25}$ The general theory is derived in Sec. II. The boundary conditions for scattering calculations are given in Sec. III, whereas some practical aspects of the calculations are discussed in Sec. IV. The results of test calculations are presented in Sec. V. The centrifugal barrier problem is considered as a model system, and the applicability of the proposed methods to realistic problems is demonstrated for cold collisions of $\mathrm{NH}$ radicals. We also calculate scattering wave functions for shape resonances in cold NH-NH collisions. Section VI contains concluding remarks.

\section{THEORY}

\section{A. Renormalized propagation}

We seek the numerical solutions of a time-independent radial Schrödinger equation

$$
\left[-\frac{\hbar^{2}}{2 \mu R} \frac{d^{2}}{d R^{2}} R+\frac{\hbar^{2} \ell(\ell+1)}{2 \mu R^{2}}+V(R)\right] \Phi(R)=E \Phi(R) .
$$

Here, $R$ is the interparticle distance, $\ell$ is the partial wave quantum number, $\mu$ is the reduced mass, $V(R)$ is the interaction, $\hbar$ $=1$ a.u. is the reduced Planck constant, and $E$ is the scattering energy. We define $\Psi(R)=R \Phi(R)$, which satisfies the simple differential equation

$$
\Psi^{\prime \prime}=W \Psi
$$

where the prime denotes differentiation with respect to $R$ and

$$
W=\frac{\ell(\ell+1)}{R^{2}}+2 \mu(V-E)
$$

First, the radial coordinate is discretized into a grid of points $\left\{R_{i}, i=0,1, \ldots, n\right\}$. The simplest method to solve this differential equation numerically, is to take this grid to be equidistant, with spacing $\Delta$, and to approximate the second derivative of the solution by finite differences

$$
\Psi_{i}^{\prime \prime}=\frac{\Psi_{i-1}-2 \Psi_{i}+\Psi_{i+1}}{\Delta^{2}}+\mathcal{O}\left(\Delta^{2}\right)=W_{i} \Psi_{i} .
$$

Here, and in the remainder of this work, subscripts denote evaluation in a grid point. This relation can be used to determine the solution in the point $R_{i+1}$, if it is known in the two previous points

$$
\Psi_{i+1}=\left(\Delta^{2} W_{i}+2\right) \Psi_{i}-\Psi_{i-1}+\mathcal{O}\left(\Delta^{4}\right) .
$$

The error that is made in each propagation step, the local error, is of order $\Delta^{4}$. However, the global convergence, the convergence as a function of the number of steps on a fixed interval, is of the order $\Delta^{2}$, and this approach is said to be a second-order algorithm. ${ }^{26}$

One can improve on this, starting from a Taylor expansion of the wave function around the center grid point, up to fifth order in $\Delta$,

$$
\begin{aligned}
\Psi_{i \pm 1}= & \Psi_{i} \pm \Delta \Psi_{i}^{\prime}+\frac{\Delta^{2}}{2} \Psi_{i}^{\prime \prime} \pm \frac{\Delta^{3}}{3 !} \Psi_{i}^{(3)} \\
& +\frac{\Delta^{4}}{4 !} \Psi_{i}^{(4)} \pm \frac{\Delta^{5}}{5 !} \Psi_{i}^{(5)}+\mathcal{O}\left(\Delta^{6}\right),
\end{aligned}
$$

with $\Psi^{(n)}$ denoting the $n$th derivative of $\Psi$ with respect to $R$. Adding these expansions for the points $R_{i+1}$ and $R_{i-1}$, all terms odd in $\Delta$ cancel exactly, and one obtains

$$
\Psi_{i+1}+\Psi_{i-1}=2 \Psi_{i}+\Delta^{2} \Psi_{i}^{\prime \prime}+\frac{\Delta^{4}}{12} \Psi_{i}^{(4)}+\mathcal{O}\left(\Delta^{6}\right) .
$$

One can solve this equation for $\Psi_{i+1}$, using

$$
\begin{aligned}
\Psi_{i}^{\prime \prime} & =W_{i} \Psi_{i}, \\
\Psi_{i}^{(4)} & =(W \Psi)_{i}^{\prime \prime}=\frac{W_{i-1} \Psi_{i-1}-2 W_{i} \Psi_{i}+W_{i+1} \Psi_{i+1}}{\Delta^{2}}+\mathcal{O}\left(\Delta^{2}\right),
\end{aligned}
$$

to obtain

$$
\begin{aligned}
\Psi_{i+1}= & \left(1-\frac{\Delta^{2} W_{i+1}}{12}\right)^{-1}\left[\Psi_{i}\left(2+\frac{5 \Delta^{2} W_{i}}{6}\right)\right. \\
& \left.-\Psi_{i-1}\left(1-\frac{\Delta^{2} W_{i-1}}{12}\right)\right]+\mathcal{O}\left(\Delta^{6}\right) .
\end{aligned}
$$

This algorithm is known as Numerov's method. ${ }^{27}$ For this algorithm, the local error is of the order $\Delta^{6}$, whereas global convergence is of the order $\Delta^{4}$, thus this method is a fourthorder algorithm.

The two methods sketched above are numerically unstable, since propagation through classically forbidden regions may lead to numerical overflow and linear dependence of the solutions. ${ }^{1,2}$ These problems are eliminated by propagating the Q-matrix, the ratio of the solution in two points, $\Psi_{i-1}$ $=Q_{i} \Psi_{i}$, rather than propagating the solution itself. In order to renormalize the methods derived above, we substitute $\Psi_{i-1}$ $=Q_{i} \Psi_{i}$, and $\Psi_{i+1}=Q_{i+1}^{-1} \Psi_{i}$ into the recurrence relations, and assume $\Psi_{i}$ to be non-zero. This yields the renormalized finite-differences method,

$$
Q_{i+1}=\left(2+\Delta^{2} W_{i}-Q_{i}\right)^{-1},
$$

and the renormalized Numerov method

$$
\begin{aligned}
Q_{i+1}= & {\left[2+\frac{10 \Delta^{2} W_{i}}{12}-\left(1-\frac{\Delta^{2} W_{i-1}}{12}\right) Q_{i}\right]^{-1} } \\
& \times\left(1-\frac{\Delta^{2} W_{i+1}}{12}\right) .
\end{aligned}
$$


To cast the above results into a more general form, we note that any second-order differential equation has two linearly independent solutions, $f(R)$ and $g(R)$. Evaluating these solutions in the three grid points provides a mapping between the solution and a point in $\mathbb{R}^{3}, f(R) \mapsto\left(f_{i-1}, f_{i}, f_{i+1}\right)$ and $g(R) \mapsto\left(g_{i-1}, g_{i}, g_{i+1}\right)$. Since all solutions are linear combinations of the two independent solutions, the solutions are associated with a plane in $\mathbb{R}^{3}$. The plane of solutions is characterized by its surface normal $\left(A^{(i)}, B^{(i)}, C^{(i)}\right)$, which can be determined as the cross product of the vectors associated with $f(R)$ and $g(R)$, as

$$
\begin{aligned}
& A^{(i)}=f_{i}^{(i)} g_{i+1}^{(i)}-f_{i+1}^{(i)} g_{i}^{(i)}, \\
& B^{(i)}=f_{i+1}^{(i)} g_{i-1}^{(i)}-f_{i-1}^{(i)} g_{i+1}^{(i)}, \\
& C^{(i)}=f_{i-1}^{(i)} g_{i}^{(i)}-f_{i}^{(i)} g_{i-1}^{(i)} .
\end{aligned}
$$

This means that one can always find $A^{(i)}, B^{(i)}$, and $C^{(i)}$, such that any solution satisfies

$$
A^{(i)} \Psi_{i-1}+B^{(i)} \Psi_{i}+C^{(i)} \Psi_{i+1}=0 .
$$

Then, one can propagate $Q$ exactly according to

$$
Q_{i+1}=-\left(A^{(i)} Q_{i}+B^{(i)}\right)^{-1} C^{(i)} .
$$

The difference between methods is how $A^{(i)}, B^{(i)}$, and $C^{(i)}$ are approximated. For the finite-differences method, Eq. (10), one has

$$
\begin{aligned}
& A^{(i)}=-1, \\
& B^{(i)}=2+\Delta^{2} W_{i}, \\
& C^{(i)}=-1,
\end{aligned}
$$

whereas the renormalized Numerov method, Eq. (11), uses

$$
\begin{aligned}
& A^{(i)}=-1+\frac{\Delta^{2} W_{i-1}}{12}, \\
& B^{(i)}=2+\frac{10 \Delta^{2} W_{i}}{12}, \\
& C^{(i)}=-1+\frac{\Delta^{2} W_{i+1}}{12} .
\end{aligned}
$$

Both the finite-differences and Numerov method are solution-following algorithms, since they approximate the solution, $\Psi$, by truncating its Taylor expansion. Therefore, these algorithms are convergent if the grid spacing, $\Delta$, is small compared to the length scale at which the solution varies. However, it may happen that the length scale at which the potential varies is in fact much larger than the length scale of the solution. In this case, it will be more efficient to approximate the potential instead. This leads to a potential-following algorithm, where one propagates in terms of the exact solutions of an approximated potential.

\section{B. Potential-following propagation}

The basic strategy to develop a renormalized potentialfollowing propagator is the following. We approximate $W(R)$, Eq. (3), on the interval $\left[R_{i-1}, R_{i+1}\right]$ by a sufficiently simple form, the reference potential, $W^{(i)}(R)$. Within this approximation, the two linearly independent solutions, $f^{(i)}(R)$ and

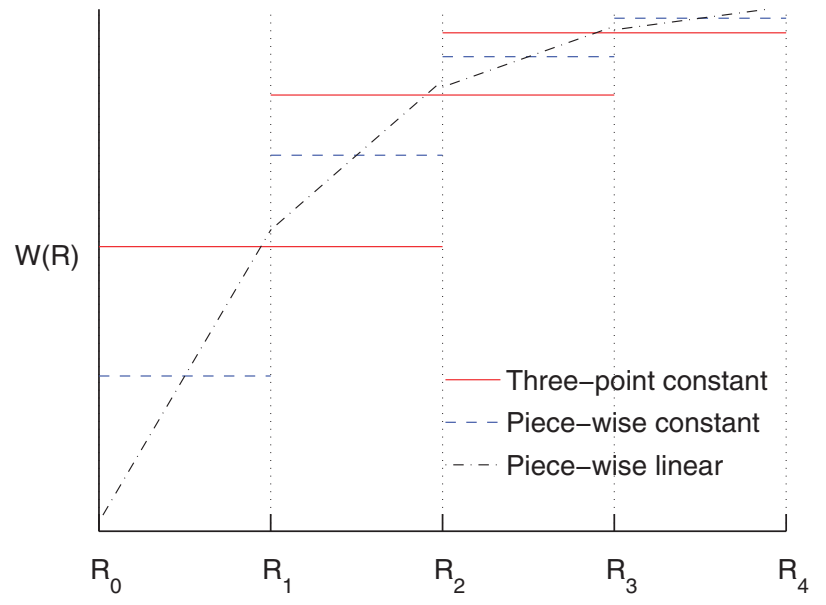

FIG. 1. Three choices of the reference potential, where the vertical dotted lines mark the radial grid points.

$g^{(i)}(R)$, are known analytically. With two independent solutions known, one may determine the plane of solutions, and propagate in terms of these solutions using Eq. (14). We stress that the reference potential also contains the collision energy and the centrifugal term, and not only the interaction, $V(R)$, as the term reference potential may suggest.

Rather than using the cross product, Eq. (12), we use

$$
\begin{aligned}
& A^{(i)}=B^{(i)} \frac{f_{i}^{(i)} g_{i+1}^{(i)}-f_{i+1}^{(i)} g_{i}^{(i)}}{f_{i+1}^{(i)} g_{i-1}^{(i)}-f_{i-1}^{(i)} g_{i+1}^{(i)}}, \\
& C^{(i)}=B^{(i)} \frac{f_{i-1}^{(i)} g_{i}^{(i)}-f_{i}^{(i)} g_{i-1}^{(i)}}{f_{i+1}^{(i)} g_{i-1}^{(i)}-f_{i-1}^{(i)} g_{i+1}^{(i)}},
\end{aligned}
$$

and take $B^{(i)}=1$. With this choice, the algorithm contains one fewer matrix multiplication, but this could in principle lead to numerical problems if $B^{(i)}=0$. However, such problems were not observed in any of the test calculations reported in Sec. V. Note that this strategy does not require one to use equidistant radial grids; that is, the step size is variable.

We use a piece-wise definition of the reference potential

$$
W^{(i)}(R)= \begin{cases}W^{(i-1, i)}(R) & \text { if } R<R_{i}, \\ W^{(i, i+1)}(R) & \text { if } R>R_{i},\end{cases}
$$

and subsequently approximate $W^{(i, i+1)}(R)$ by a simple analytical form for each unique two-point interval. Explicitly, we will consider the piece-wise constant and piece-wise linear approximations. The definitions of these reference potentials are illustrated in Fig. 1. The analytic solutions for constant and linear reference potentials are the trigonometric and Airy functions, respectively, and the algorithms are expected to be second and fourth order. ${ }^{8}$ In Appendix A, we treat the evaluation of $A^{(i)}, B^{(i)}$, and $C^{(i)}$ for piece-wise defined reference potentials, and list explicit expressions for the piece-wise constant and linear reference potentials.

One can think of a reference potential which is defined on each three-point interval, rather than on each two-point interval. However, such reference potentials are not singlevalued, as is illustrated for a three-point constant reference potential in Fig. 1. In the case of an equidistant radial grid, 
the three-point constant reference potential algorithm reduces to the enhanced renormalized Numerov method of Thorlacius and Cooper, ${ }^{9}$ as is shown in Appendix B.

\section{Multichannel case}

Here, we generalize the methods to the multichannel case, where the time-independent Schrödinger equation takes the form

$$
\begin{aligned}
& {\left[-\frac{\hbar^{2}}{2 \mu R} \frac{d^{2}}{d R^{2}} R+\frac{\hat{\ell}^{2}}{2 \mu R^{2}}+\hat{V}(R, \omega)+\hat{H}_{\text {asymp }}(\omega)\right] \Phi(R, \omega)} \\
& \quad=E \Phi(R, \omega)
\end{aligned}
$$

The interaction, $\hat{V}$, depends on the separation, $R$, and all remaining coordinates, $\omega$, and it vanishes for $R \rightarrow \infty$. The sum of the Hamiltonians of the fragments is denoted by $\hat{H}_{\text {asymp }}$. The wave function is expanded in a set of channel functions, $\left\{\phi_{p}(\omega), p=1,2, \ldots, N\right\}$, as

$$
\Phi_{q}(R, \omega)=R^{-1} \sum_{p=1}^{N} \phi_{p}(\omega) \Psi_{p q}(R),
$$

where $q$ labels the independent solutions as discussed in Sec. III. In practice, the channel basis is often truncated, see Sec. V B for an example. The matrix of expansion coefficients, $\boldsymbol{\Psi}$, satisfies the set of coupled second-order differential equations

$$
\Psi^{\prime \prime}=W \Psi
$$

Here, $\boldsymbol{W}$ is a Hermitian matrix, with elements

$$
W_{p q}=2 \mu\left\langle\phi_{p}\left|\frac{\hat{\ell}^{2}}{2 \mu R^{2}}+\hat{V}+\hat{H}_{\text {asymp }}-E\right| \phi_{q}\right\rangle
$$

Again, the radial coordinate is discretized into a grid of points $\left\{R_{i}, i=0,1, \ldots, n\right\}$, and Eqs. (13) and (14) are generalized to

$$
\begin{gathered}
\boldsymbol{A}^{(i)} \boldsymbol{\Psi}_{i-1}+\boldsymbol{B}^{(i)} \boldsymbol{\Psi}_{i}+\boldsymbol{C}^{(i)} \boldsymbol{\Psi}_{i+1}=0, \\
\boldsymbol{Q}_{i+1}=-\left(\boldsymbol{A}^{(i)} \boldsymbol{Q}_{i}+\boldsymbol{B}^{(i)}\right)^{-1} \boldsymbol{C}^{(i)} .
\end{gathered}
$$

Next, we employ a locally adiabatic approximation. ${ }^{8}$ That is, we diagonalize the $\mathrm{W}$-matrix with a unitary transformation,

$$
\widetilde{\boldsymbol{W}}_{n}^{(i)}=\boldsymbol{U}^{(i) \dagger} \boldsymbol{W}_{n}^{(i)} \boldsymbol{U}^{(i)},
$$

and we assume this transformation, $\boldsymbol{U}^{(i)}$, to be constant over the three-point interval ranging from $R_{i-1}$ to $R_{i+1}$. That is, the W-matrix is diagonal in the locally adiabatic basis $\left\{\chi_{p}^{(i)}(\omega)\right\}$, where the $p$ th basis function, $\chi_{p}^{(i)}(\omega)$, can be expressed in the primitive basis, $\left\{\phi_{p}(\omega)\right\}$, as

$$
\chi_{p}^{(i)}(\omega)=\sum_{r=1}^{N} \phi_{r}(\omega) U_{r p}^{(i)}
$$

We denote the remaining relevant quantities in the basis $\left\{\chi_{p}^{(i)}(\omega)\right\}$ as

$$
\begin{aligned}
& \widetilde{\boldsymbol{A}}^{(i)}=\boldsymbol{U}^{(i) \dagger} \boldsymbol{A}^{(i)} \boldsymbol{U}^{(i)}, \\
& \widetilde{\boldsymbol{B}}^{(i)}=\boldsymbol{U}^{(i) \dagger} \boldsymbol{B}^{(i)} \boldsymbol{U}^{(i)}, \\
& \widetilde{\boldsymbol{C}}^{(i)}=\boldsymbol{U}^{(i) \dagger} \boldsymbol{C}^{(i)} \boldsymbol{U}^{(i)}, \\
& \widetilde{\boldsymbol{f}}_{j}^{(i)}=\boldsymbol{U}^{(i) \dagger} \boldsymbol{f}_{j}^{(i)}, \\
& \widetilde{\boldsymbol{g}}_{j}^{(i)}=\boldsymbol{U}^{(i) \dagger} \boldsymbol{g}_{j}^{(i)}, \\
& \widetilde{\boldsymbol{\Psi}}_{j}^{(i)}=\boldsymbol{U}^{(i) \dagger} \boldsymbol{\Psi}_{j},
\end{aligned}
$$

where $j \in\{i-1, i, i+1\}$.

In the locally adiabatic basis, $\left\{\chi_{p}^{(i)}(\omega)\right\}$, the W-matrix is diagonal, and the differential equations decouple. Therefore, the problem reduces to a series of single-channel problems. This means that the matrices $\widetilde{\boldsymbol{f}}_{j}^{(i)}, \widetilde{\boldsymbol{g}}_{j}^{(i)}, \widetilde{\boldsymbol{A}}^{(i)}, \widetilde{\boldsymbol{B}}^{(i)}$, and $\widetilde{\boldsymbol{C}}^{(i)}$ are diagonal, and the diagonal elements can be determined as for the single-channel case.

Each adiabatic potential, corresponding to a diagonal element of the W-matrix, $\widetilde{W}_{a, a}^{(i)}(R)$, is approximated by a simple reference potential. Denoting the independent solutions for adiabat $a$ on interval $i$ as $f^{(i, a)}(R)$ and $g^{(i, a)}(R)$, we have for the diagonal elements of $\widetilde{\boldsymbol{A}}^{(i)}, \widetilde{\boldsymbol{B}}^{(i)}$, and $\widetilde{\boldsymbol{C}}^{(i)}$,

$$
\begin{aligned}
\widetilde{A}_{a, a}^{(i)} & =\frac{f_{i}^{(i, a)} g_{i+1}^{(i, a)}-f_{i+1}^{(i, a)} g_{i}^{(i, a)}}{f_{i+1}^{(i, a)} g_{i-1}^{(i, a)}-f_{i-1}^{(i, a)} g_{i+1}^{(i, a)},} \\
\widetilde{B}_{a, a}^{(i)} & =1, \\
\widetilde{C}_{a, a}^{(i)} & =\frac{f_{i-1}^{(i, a)} g_{i}^{(i, a)}-f_{i}^{(i, a)} g_{i-1}^{(i, a)}}{f_{i+1}^{(i, a)} g_{i-1}^{(i, a)}-f_{i-1}^{(i, a)} g_{i+1}^{(i, a)}} .
\end{aligned}
$$

Explicit expressions for $\widetilde{A}_{a, a}^{(i)}$ and $\widetilde{C}_{a, a}^{(i)}$ are found in Appendix A for piece-wise constant and linear reference potentials. Finally, we transform back to the primitive basis, $\left\{\phi_{p}(\omega)\right\}$, using the inverse of Eq. (26),

$$
\begin{aligned}
& \boldsymbol{A}^{(i)}=\boldsymbol{U}^{(i)} \widetilde{\boldsymbol{A}}^{(i)} \boldsymbol{U}^{(i) \dagger}, \\
& \boldsymbol{B}^{(i)}=\mathbf{1}, \\
& \boldsymbol{C}^{(i)}=\boldsymbol{U}^{(i)} \widetilde{\boldsymbol{C}}^{(i)} \boldsymbol{U}^{(i) \dagger},
\end{aligned}
$$

and propagate the Q-matrix according to Eq. (23).

Since the renormalized propagation algorithm propagates across three-point intervals, there is a subtlety involving the locally adiabatic approximation. Each two-point interval occurs in two successive propagation steps. In each of these steps, the potential is approximated differently, as it is assumed to be diagonalized by a transformation that depends on the three-point interval, Eq. (24). In potential-following log-derivative propagation, one propagates across two-point intervals, ${ }^{8}$ such that this issue is never encountered. In numerical applications presented in Sec. V, we find that this issue does not affect the accuracy, as log-derivative and renormalized algorithms with the same reference potential are equally accurate. 


\section{BOUNDARY CONDITIONS}

The solution of the time-independent Schrödinger equation depends on the boundary conditions, which are given in this section. The requirement that the wave function, $\Phi(R)$, is finite at $R=0$ yields

$$
\boldsymbol{\Psi}(R=0)=\mathbf{0} .
$$

This leads to the boundary condition $\boldsymbol{Q}_{1}=\mathbf{0}$. In practical applications, one may impose the same boundary conditions for some $R_{0}>0$, if $R_{0}$ is sufficiently small such that the potential is strongly repulsive and one may assume the wave function to vanish.

At asymptotically large $R$, one may impose the scattering boundary conditions

$$
\Phi_{q}(R, \omega) \sim \sum_{p} \varphi_{p}(\omega)\left[h_{\ell_{p}}^{(2)}\left(k_{p} R\right) \delta_{p q}+h_{\ell_{p}}^{(1)}\left(k_{p} R\right) S_{p q}\right],
$$

where $\left\{\varphi_{p}(\omega)\right\}$ is a basis of simultaneous eigenfunctions of $\hat{H}_{\text {asymp }}$ and $\hat{\ell}^{2}$, with eigenvalues $\epsilon_{p}$ and $\hbar^{2} \ell_{p}\left(\ell_{p}+1\right)$, respectively. The spherical Hankel functions of the first and second kind are denoted $h_{\ell}^{(1)}(x)$ and $h_{\ell}^{(2)}(x),{ }^{28}$ and the Kronecker delta is given by

$$
\delta_{p q}=\left\{\begin{array}{ll}
1 & \text { if } p=q \\
0 & \text { if } p \neq q
\end{array} .\right.
$$

The wave vectors are given by $k_{p}=\frac{1}{\hbar} \sqrt{2 \mu\left(E-\epsilon_{p}\right)}$. The elements of the unitary scattering matrix (S-matrix) are denoted $S_{p q}$. The S-matrix usually is the quantity one is interested in, as observables such as collision cross sections and rate constants can be determined from its elements.

The S-matrix is determined from the Q-matrix in the last gridpoint, $R_{n}{ }^{2}$ First, the Q-matrix is transformed to the asymptotic basis, using

$$
\begin{aligned}
\tilde{\mathbf{Q}}_{n} & =\mathbf{U}^{\dagger} \mathbf{Q}_{n} \mathbf{U}, \\
U_{p q} & =\left\langle\phi_{p} \mid \varphi_{q}\right\rangle .
\end{aligned}
$$

We determine the K-matrix as

$$
\mathbf{K}=\left(\mathbf{G}_{n-1}-\tilde{\mathbf{Q}}_{n} \mathbf{G}_{n}\right)^{-1}\left(\mathbf{F}_{n-1}-\tilde{\mathbf{Q}}_{n} \mathbf{F}_{n}\right) .
$$

In the above, $\mathbf{F}_{i}$ and $\mathbf{G}_{i}$ are diagonal matrices, whose elements are related to the spherical Bessel functions for open channels

$$
\begin{aligned}
\left(\mathbf{F}_{n}\right)_{i j} & =\delta_{i j} k_{i}^{1 / 2} R j_{\ell_{i}}\left(k_{i} R_{n}\right), \\
(\mathbf{G})_{i j} & =\delta_{i j} k_{i}^{1 / 2} R y_{\ell_{i}}\left(k_{i} R_{n}\right),
\end{aligned}
$$

and modified Bessel functions for the closed channels

$$
\begin{aligned}
& \left(\mathbf{F}_{n}\right)_{i j}=\delta_{i j}\left(k_{i} R\right)^{1 / 2} I_{\ell_{i}+1 / 2}\left(k_{i} R_{n}\right), \\
& \left(\mathbf{G}_{n}\right)_{i j}=\delta_{i j}\left(k_{i} R\right)^{1 / 2} K_{\ell_{i}+1 / 2}\left(k_{i} R_{n}\right) .
\end{aligned}
$$

The S-matrix is then determined from the open-open block of the K-matrix, $\tilde{\mathbf{K}}$, as

$$
\mathbf{S}=(\mathbf{1}-i \tilde{\mathbf{K}})^{-1}(\mathbf{1}+i \tilde{\mathbf{K}}),
$$

with $\mathbf{1}$ a unit matrix and $i$ the imaginary unit.

Finally, we note that one can also impose reactive boundary conditions, where flux is allowed to escape in to reactive channels at short range. ${ }^{19,29}$ The developed methods can be used with these boundary conditions, but we will not consider such calculations in this paper.

\section{PRACTICAL CONSIDERATIONS}

Potential-following propagation is efficient if the potential varies slowly. In the short range of the potential, this will not be the case, and solution-following methods such as renormalized Numerov may be more efficient. These two methods may conveniently be combined, resulting in a hybrid algorithm, which is efficient in both regimes. For the implementation, this means that the first Q-matrix, $Q_{i}$ in Eq. (23), is determined by a solution-following method, rather than by the boundary conditions, Eq. (29).

The performance of a potential-following method depends not only on the functional form of the reference potential, but also on how its parameters are chosen in each interval. As argued by Alexander and Gordon, for a linear reference potential the optimal choice may be found by using the exact potential at the two-point Gauss-Legendre abscissae. ${ }^{30}$ For constant reference potentials, we use the exact potential at the center of the interval.

In practical applications, matrix operations form the computational bottleneck, as the required computational time scales with the third power of the number of channels. In this context, it is interesting to note that the locally adiabatic basis is independent of the scattering energy, which only shifts the diagonal of the W-matrix. Therefore, the diagonalization of the W-matrix does not have to be performed repeatedly for successive energies, as long as computer memory permits the transformation to the adiabatic basis to be stored.

The other required matrix operations are multiplications and inversions. Our algorithm requires three matrix multiplications and one matrix inverse per propagation step. We note that an equivalent algorithm can be derived by propagating in the adiabatic basis, as is done in Ref. 10. By storing the energy-independent overlap matrices,

$$
\boldsymbol{O}_{i, i+1}=\boldsymbol{U}^{(i) \dagger} \boldsymbol{U}^{(i+1)},
$$

and propagating a transformed Q-matrix, one can save two matrix multiplications per step. ${ }^{10}$ In this case, the computational cost per propagation step is identical to that of renormalized Numerov. ${ }^{1,2,10}$

\section{RESULTS}

In what follows, we use the following designation for the different algorithms. The renormalized algorithms with piecewise constant and linear reference potentials are denoted $Q$ sin and Qairy, respectively. The names refer to the linearly independent solutions, the trigonometric and Airy functions. 
These methods are compared to existing log-derivative propagators, which use the same reference potentials. These are denoted Ysin and Yairy. ${ }^{8}$

Implementations of Qsin and Qairy can be found in the supplementary material, ${ }^{25}$ along with test programs for constant and linear potentials. Also included are an implementation of the renormalized Numerov algorithm, ${ }^{1,2}$ and a physical test problem, describing the collinear collision of a hydrogen molecule with a helium atom. ${ }^{31}$ The programs are available for Scilab, ${ }^{32}$ and the commercial alternative Matlab. $^{33}$

First of all, it has been verified that the renormalized potential-following algorithms yield the exact Q-matrix if the potential equals the reference potential. The corresponding log-derivative algorithms yield the numerically exact Y-matrix for the approximated potentials. The $Q \sin$ and $Y$ sin algorithms are equivalent in the sense that they both yield the exact solution for the same approximated potential, the piece-wise constant reference potential. The same holds for Qairy and Yairy, which use the same piece-wise linear reference potential. We have applied our new algorithms to both a single-channel and a multichannel scattering problem, as described below.

\section{A. Centrifugal barrier}

A particularly convenient model system is given by the single-channel problem in which the potential is given by the centrifugal barrier only. That is, we take the Schrödinger equation, Eq. (1), with $V(R)=0$. The potential is physically relevant, as it is the asymptotic form of any potential involving at most one charged particle. Furthermore, analytical solutions in terms of spherical Bessel functions are known, thus allowing for a rigorous test of our numerical results. Since it is a single-channel problem, the locally adiabatic basis is not an approximation. Hence, this calculation only tests the efficiency of the reference potentials.

As a test case, we consider the $d$-wave scattering of two nitrogen atoms at a collision energy of $100 \mathrm{~cm}^{-1}$. Thus, the centrifugal barrier corresponds to $\ell=2$ and the reduced mass is $\mu=\mu_{N} / 2$ with $\mu_{N}$ the mass of a ${ }^{14} \mathrm{~N}$ nucleus. Figure 2 shows the convergence of the S-matrix, as a function of the number of steps on equidistant and logarithmically scaled grids on the interval $0.001 a_{0}$ to $100.001 a_{0}$. The error is defined as the norm of the difference between the obtained and the exact S-matrix. The results of the constant reference potential algorithms, $Q \sin$ and $Y \sin$, which are equivalent as argued above, match closely. The same is true for the linear reference potential algorithms, Qairy and Yairy. On an equidistant grid, the error of the second-order algorithm $Q \sin$ varies as $\Delta^{-2}$. Similarly, the error for the fourth-order algorithm Qairy varies as $\Delta^{-4}$. On a logarithmically scaled grid, we reach the same accuracy with an order of magnitude fewer grid points, clearly demonstrating the advantage of a variable step size.

\section{B. $\mathrm{NH}-\mathrm{NH}$}

To test the applicability of the reported methods to realistic multichannel problems, we consider the scattering of

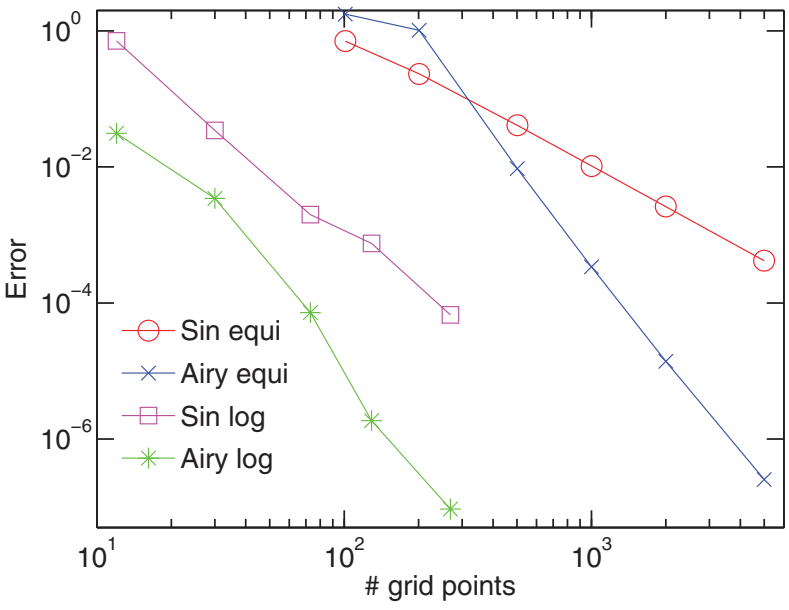

FIG. 2. Error in the S-matrix for the centrifugal barrier problem, as a function of the number of steps on equidistant or logarithmically scaled grids. The difference between renormalized and log-derivative algorithms is not noticeable, hence we have dropped the labels $Q$ and $Y$.

two ${ }^{15} \mathrm{NH}$ radicals in field-free space at low energy. The longranged dipole-dipole interactions combined with the low energy require propagation to large separation. In literature, this problem has been treated with the Yairy algorithm, combined with a short-range propagator, using the implementations available in the Molscat package. ${ }^{3}$ In this calculation, the $\mathrm{NH}$ monomers are treated as rigid rotors, and the channel basis functions are labeled as $\left|\left(j_{A} j_{B}\right) j_{A B} \ell ; J M\right\rangle$, where $j_{X}$ is the total angular momentum of monomer $X(X=A, B)$, obtained by coupling of the rotational $\left(N_{X}\right)$ and spin $\left(S_{X}\right)$ angular momenta, $j_{A B}$ is the coupled angular momentum of $j_{A}$ and $j_{B}, J$ is the total angular momentum of the collision complex, obtained by angular-momentum coupling of $j_{A B}$ and partial wave $\ell$, and $M$ is the projection of $J$ onto the space-fixed quantization axis. The NH-NH scattering dynamics is assumed to take place on a single potential-energy surface that corresponds to total spin quantum number $S=2$. For a more detailed description of the calculation, the reader is referred to the original articles. $^{18,19}$

We have repeated these calculations with the $Q \sin$ and Qairy algorithms, combined with renormalized Numerov in the short range. ${ }^{1,2}$ The short-range algorithm is used on the interval 4.5-15 $a_{0}$, and the potential-following algorithms are used from 15 to $100 a_{0}$, see Sec. III for a discussion of the boundary conditions. The same radial grids were used for renormalized propagation and for propagation with the Yairy algorithm in Molscat. Here, the channel basis was truncated at partial wave quantum number $\ell_{\max }=4$ and monomer rotational quantum number $N_{\max }=2$, and the calculation was performed for $J=2$ at an energy of $1 \mathrm{~K}$. For odd parity and permutation symmetry, the calculation thus involves 119 coupled channels.

The convergence of the S-matrix is shown in Fig. 3. Here, we define the error as the norm of the difference between the current and most accurately calculated S-matrix. The norm of the matrix is defined as the largest singular value. The figure also includes the convergence for the renormalized $\mathrm{Nu}$ merov method. ${ }^{1,2}$ This method requires an order of magnitude 


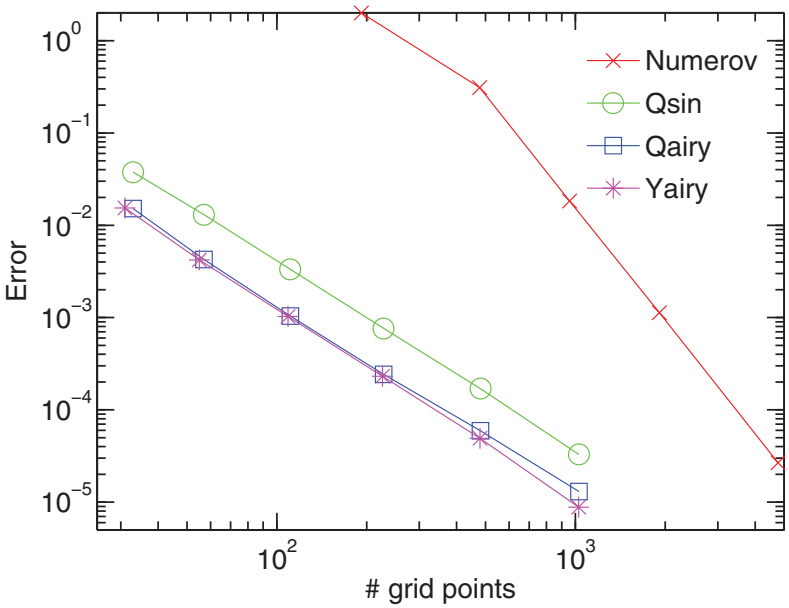

FIG. 3. Error in the S-matrix for NH-NH collisions at $E=1 \mathrm{~K}$, for odd parity and permutation symmetry, and total angular momentum $J=2$, as a function of the number of propagation steps in the long range. The error is defined in the main text. The radial grid for the potential-following propagators was generated by Molscat, whereas renormalized Numerov uses an equidistant grid.

more grid points to achieve reasonable convergence, thus illustrating the advantage of potential-following over solutionfollowing methods in the long range.

It can be seen that the two types of Airy propagators give almost identical errors, and for both algorithms the convergence of the S-matrix is second order in the number of grid points. The reason that convergence is no longer fourth order seems to be that it is the locally adiabatic approximation that is limiting the accuracy. The first-derivative matrix is not diagonal in the locally adiabatic basis, and consideration of its diagonal elements by the Airy propagators constitutes little improvement. For practical applications to systems such as $\mathrm{NH}-\mathrm{NH}$, constant reference potential algorithms, such as Qsin, appear to be almost as accurate. The obvious advantage of the constant reference potential algorithm is that it is much simpler to implement.

An advantage of the renormalized algorithms is that the Q-matrices they provide, allow for straightforward calculation of the scattering wave function. To illustrate this, we show the wave function for scattering from the $\left|\left(j_{A} j_{B}\right) j_{A B} \ell ; J M\right\rangle$ $=|(11) 21 ; 22\rangle$ channel in Fig. 4, where each line corresponds to a different channel. The entrance channel is shown as the blue line which asymptotically has large amplitude. This figure also shows the scattering wave function, obtained after scaling the reduced mass by $2.2 \%$. These $\lambda$-scans are used to sample the sensitivity of the cross sections to scattering resonances. With this scaling we find a shape resonance, i.e., a quasi-bound state trapped behind the centrifugal barrier. This results in a scattering wave function with a large amplitude at short range, as can be seen in Fig. 4.

Besides allowing one to analyze scattering resonances, the reconstruction of scattering wave functions allows one to calculate matrix elements over continuum states. This means the methods developed can be used to describe photodissociation and photoassociation. ${ }^{24}$ It is also possible to directly propagate matrix elements over the scattering states, without their explicit reconstruction on the complete radial grid. ${ }^{5}$
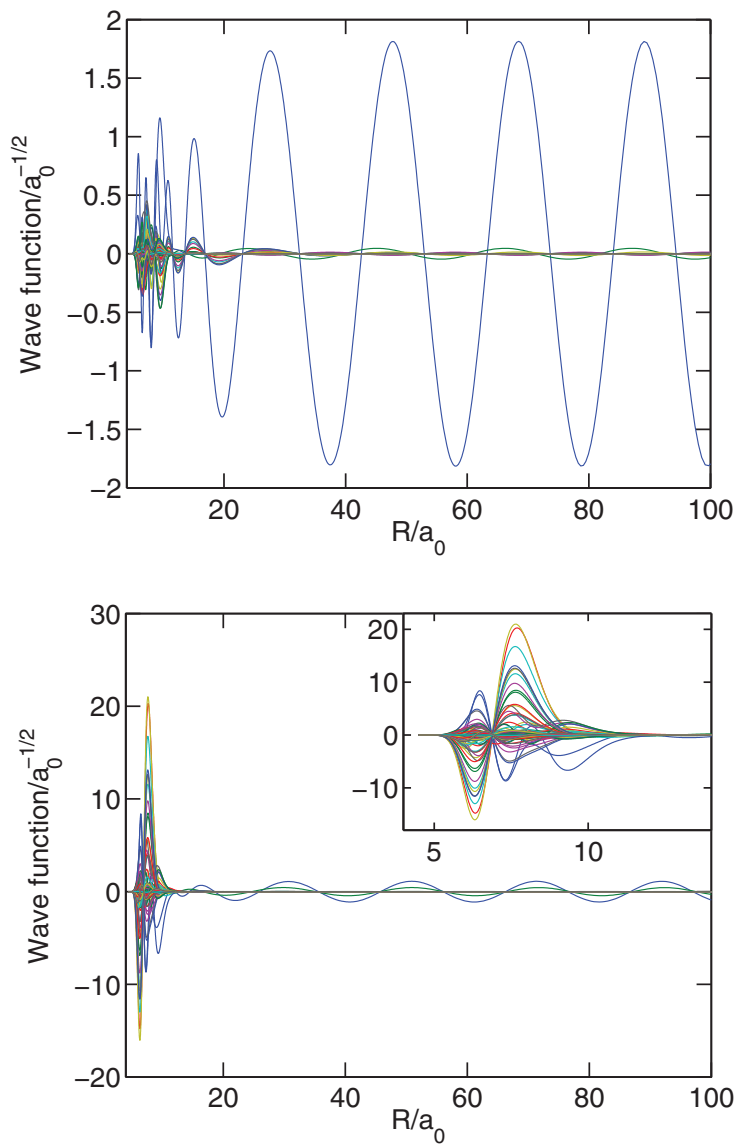

FIG. 4. The upper panel shows the scattering wave function for NH-NH collisions from a $\left|\left(j_{A} j_{B}\right) j_{A B} \ell ; J M\right\rangle=|(11) 21 ; 22\rangle$ entrance channel at an energy of $1 \mathrm{~K}$. The lines correspond to different channels. The blue line which asymptotically has large amplitude corresponds to the entrance channel. The lower panel shows the wave function for a shape resonance in the same channel, as found after scaling the reduced mass by $2.2 \%$.

\section{CONCLUSION}

We have presented a general theory for renormalized potential-following propagation, and explicitly consider piece-wise constant and piece-wise linear reference potentials. The applicability of these algorithms to realistic problems has been demonstrated for cold NH-NH collisions. In such applications, the accuracy is determined by the locally adiabatic approximation, rather than by the form of reference potential. Therefore, the constant reference potential algorithm, which is simple to implement, yields almost the same accuracy as the linear reference potential method.

The potential-following algorithms, derived in this work, are especially efficient for problems where one has to propagate to very large distances, e.g., cold and ultracold collisions of species with long-ranged interactions. The step size is variable, the method is compatible with reactive boundary conditions, and the algorithm may be combined with renormalized Numerov in the short range. The renormalized approach, opposed to log-derivative propagation, allows one to reconstruct scattering wave functions in a straightforward way, and therefore to describe near-threshold photodissociation and photoassociation of ultracold atoms. 


\section{ACKNOWLEDGMENTS}

The authors thank Ad van der Avoird for carefully reading the paper.

\section{APPENDIX A: NUMERICAL EVALUATION}

Numerical evaluation of Eq. (27) for the considered reference potentials may be difficult. Problems occur especially for the linear reference potential in the case of a vanishing slope. In this case, the arguments of the Airy functions become increasingly large and more closely spaced. This invites for evaluation through the known asymptotic expansion of the Airy functions, ${ }^{28}$ but this is not straightforward since we are considering a sector composed of two different linear intervals. In this appendix, we therefore express Eq. (27) as much as possible in terms of factors depending on the solutions on each two-point interval. Next, we give explicit expressions for the constant reference potential in Appendix A 1, and give an asymptotic expansion for the linear reference potential in Appendix A 2. The definitions of the reference potentials, their linearly independent solutions, and the corresponding Wronskians, $\mathcal{W}=f g^{\prime}-f^{\prime} g$, are listed in Table I. For notational ease, we will suppress the tildes denoting that we are considering a locally adiabatic potential, as well as superscripts $i$ and $a$, denoting the three-point interval and adiabat, respectively.

On each three-point interval, we consider two segments, ranging from the grid-points $R_{i-1}$ to $R_{i}$ and $R_{i}$ to $R_{i+1}$, respectively. On these two-point intervals, a certain reference potential is assumed, and within this approximation, the linearly independent solutions on each interval are known. The solutions are denoted $a^{(j)}, b^{(j)}$, where $j=1,2$ labels the interval. The linearly independent solutions on the sector consisting of two such intervals are given by

$$
\begin{aligned}
& f(R)= \begin{cases}a^{(1)}(R) & {\left[R_{n-1}, R_{n}\right],} \\
c_{a} a^{(2)}(R)+c_{b} b^{(2)}(R) & {\left[R_{n}, R_{n+1}\right],}\end{cases} \\
& g(R)= \begin{cases}b^{(1)}(R) & {\left[R_{n-1}, R_{n}\right],} \\
d_{a} a^{(2)}(R)+d_{b} b^{(2)}(R) & {\left[R_{n}, R_{n+1}\right]}\end{cases}
\end{aligned}
$$

The coefficients $c_{a}, c_{b}, d_{a}$, and $d_{b}$ are found by requiring $f, f$, $g$, and $g^{\prime}$ to be continuous at $R_{n}$. This gives

$$
\left(\begin{array}{c}
c_{a} \\
c_{b}
\end{array}\right)=\frac{1}{a_{n}^{(2)} b_{n}^{(2) \prime}-a_{n}^{(2) \prime} b_{n}^{(2)}}\left(\begin{array}{cc}
b_{n}^{(2) \prime} & -b_{n}^{(2)} \\
-a_{n}^{(2) \prime} & a_{n}^{(2)}
\end{array}\right)\left(\begin{array}{c}
a_{n}^{(1)} \\
a_{n}^{(1) \prime}
\end{array}\right),
$$

and analogously for $d_{a}$ and $d_{b}$.
To evaluate Eq. (27), we consider its numerator and denominator separately. For the numerator there are two cases, corresponding to $A^{(i)}$ and $C^{(i)}$. For the numerator in the expression for $C^{(i)}$ we find

$$
f_{n} g_{n-1}-f_{n-1} g_{n}=X_{1}^{-},
$$

and for $A^{(i)}$

$$
f_{n} g_{n+1}-f_{n+1} g_{n}=\left(c_{a} d_{b}-c_{b} d_{a}\right) X_{2}^{+},
$$

where we have defined

$$
X_{j}^{ \pm}=a_{i}^{(j)} b_{i \pm 1}^{(j)}-a_{i \pm 1}^{(j)} b_{i}^{(j)},
$$

where $n$ is the center grid point of the three-point interval. Evaluation of $X_{j}^{ \pm}$is numerically safe, as will be shown below. The quantity in parentheses can be related to the Wronskians of the solutions on both intervals as

$$
c_{a} d_{b}-c_{b} d_{a}=\frac{\mathcal{W}^{(1)}}{\mathcal{W}^{(2)}} .
$$

Evaluation of the denominator in Eq. (27) seems less straightforward, as the functions involved are defined on different intervals. Upon insertion of the definitions (A2) and analogues for $d_{a, b}$, one can express the denominator in terms of factors involving the independent solutions on the same interval. The result is given by

$$
f_{n+1} g_{n-1}-f_{n-1} g_{n+1}=\frac{1}{\mathcal{W}^{(2)}}\left(X_{2}^{+} Y_{1}^{-}-X_{1}^{-} Y_{2}^{+}\right),
$$

where we have defined

$$
Y_{j}^{ \pm}=a_{i}^{(j) \prime} b_{i \pm 1}^{(j)}-a_{i \pm 1}^{(j)} b_{i}^{(j) \prime}
$$

\begin{tabular}{|c|c|c|c|}
\hline Reference & Functional form & Independent solutions & Wronskian \\
\hline Constant & $W(R)=W$ & $a(R)= \begin{cases}\sin (\sqrt{|W|} R) & W<0 \\
\sinh (\sqrt{W} R) & W>0\end{cases}$ & $\mathcal{W}=-\sqrt{|W|}$ \\
\hline Linear & $W(R)=W_{0}+W_{1}\left(R-R_{i}\right)$ & $\begin{array}{c}b(R)= \begin{cases}\cos (\sqrt{|W|} R) & W<0 \\
\cosh (\sqrt{W} R) & W>0\end{cases} \\
a(R)=\mathrm{Ai}\left[W_{1}^{1 / 3}\left(W_{0} / W_{1}+\left(R-R_{i}\right)\right)\right] \\
b(R)=\operatorname{Bi}\left[W_{1}^{1 / 3}\left(W_{0} / W_{1}+\left(R-R_{i}\right)\right)\right]\end{array}$ & $\mathcal{W}=\pi^{-1} \sqrt[3]{W_{1}}$ \\
\hline
\end{tabular}

again with $n$ the center grid point.

To be explicit, the diagonal elements of the required matrices $\widetilde{\boldsymbol{A}}^{(i)}$ and $\widetilde{\boldsymbol{C}}^{(i)}$ are computed as

$$
\begin{aligned}
\widetilde{A}_{a, a}^{(n)} & =\mathcal{W}^{(1)} \frac{X_{2}^{+}}{X_{2}^{+} Y_{1}^{-}-X_{1}^{-} Y_{2}^{+}}, \\
\widetilde{C}_{a, a}^{(n)} & =\mathcal{W}^{(2)} \frac{X_{1}^{-}}{X_{1}^{-} Y_{2}^{+}-X_{2}^{+} Y_{1}^{-}},
\end{aligned}
$$

where the dependence on $a$ and $n$, enumerating the adiabats and three-point intervals, is implicit on the right-hand side.

TABLE I. Definition of constant and linear reference potential and their solutions. 


\section{Constant reference potential: Qsin}

For the constant reference potential, evaluation of Eqs. (A5) and (A8) is straightforward. For open channels, we find

$$
\begin{aligned}
& X_{j}^{ \pm}=\sin \left(\sqrt{\left|W^{(j)}\right|}\left(R_{i}-R_{i \pm 1}\right)\right), \\
& Y_{j}^{ \pm}=\sqrt{\left|W^{(j)}\right|} \cos \left(\sqrt{\left|W^{(j)}\right|}\left(R_{i}-R_{i \pm 1}\right)\right),
\end{aligned}
$$

and for closed channels we have

$$
\begin{aligned}
& X_{j}^{ \pm}=\sinh \left(\sqrt{W^{(j)}}\left(R_{i}-R_{i \pm 1}\right)\right), \\
& Y_{j}^{ \pm}=\sqrt{W^{(j)}} \cosh \left(\sqrt{W^{(j)}}\left(R_{i}-R_{i \pm 1}\right)\right) .
\end{aligned}
$$

\section{Linear reference potential: Qairy}

For the linear reference potential, Eqs. (A5) and (A8) can be evaluated numerically using routines by Amos, ${ }^{34}$ for small arguments of the Airy functions. However, the argument will become asymptotically large in the long range, as the slope of the potential tends to zero. Therefore, we will evaluate Eqs. (A5) and (A8) using the asymptotic expansion of the Airy functions. ${ }^{28}$ Defining $\Delta=W_{1}^{1 / 3}\left(R_{i \pm 1}-R_{i}\right), x=W_{0} W_{1}^{-2 / 3}$, $z=|x|, \zeta=\frac{2}{3} z^{3 / 2}, c_{k}=\frac{\Gamma(3 k+1 / 2)}{54^{k} k ! \Gamma(k+1 / 2)}$, and $d_{k}=-\frac{6 k+1}{6 k-1} c_{k}$, the asymptotic expansion for $|\Delta|$ small compared to $z$ yields

$$
\begin{aligned}
X_{j}^{ \pm}= & \frac{1}{2 \pi} z^{-1 / 2}(1+\Delta / z)^{-1 / 4} \\
& \left\{\exp \left\{\left[(1+\Delta / z)^{3 / 2}-1\right] \zeta\right\}\left(\sum_{k}(-1)^{k} c_{k} \zeta^{-k}\right)\right. \\
& \left(\sum_{k} c_{k} \zeta^{-k}(1+\Delta / z)^{-3 k / 2}\right) \\
& -\exp \left\{-\left[(1+\Delta / z)^{3 / 2}-1\right] \zeta\right\}\left(\sum_{k} c_{k} \zeta^{-k}\right) \\
& \left.\left(\sum_{k}(-1)^{k} c_{k} \zeta^{-k}(1+\Delta / z)^{-3 k / 2}\right)\right\},
\end{aligned}
$$

for positive arguments of the Airy functions, and

$$
\begin{aligned}
X_{j}^{ \pm}= & \frac{1}{\pi} z^{-1 / 2}(1-\Delta / z)^{-1 / 4} \\
& \left\{-\sin \left\{\left[(1-\Delta / z)^{3 / 2}-1\right] \zeta\right\}\right. \\
& {\left[\left(\sum_{k}(-1)^{k} c_{2 k+1} \zeta^{-(2 k+1)}(1-\Delta / z)^{-3(2 k+1) / 2}\right)\right.} \\
& \left(\sum_{k}(-1)^{k} c_{2 k+1} \zeta^{-(2 k+1)}\right)+\left(\sum_{k}(-1)^{k} c_{2 k} \zeta^{-2 k}\right) \\
& \left.\left(\sum_{k}(-1)^{k} c_{2 k} \zeta^{-2 k}(1-\Delta / z)^{-3 k}\right)\right] \\
& +\cos \left\{\left[(1-\Delta / z)^{3 / 2}-1\right] \zeta\right\} \\
& {\left[\left(\sum_{k}(-1)^{k} c_{2 k+1} \zeta^{-(2 k+1)}(1-\Delta / z)^{-3(2 k+1) / 2}\right)\right.}
\end{aligned}
$$

$$
\begin{aligned}
& \left(\sum_{k}(-1)^{k} c_{2 k} \zeta^{-2 k}\right)-\left(\sum_{k}(-1)^{k} c_{2 k+1} \zeta^{-(2 k+1)}\right) \\
& \left.\left.\left(\sum_{k}(-1)^{k} c_{2 k} \zeta^{-2 k}(1-\Delta / z)^{-3 k}\right)\right]\right\},
\end{aligned}
$$

for negative arguments.

Similarly, we find expansions for $Y_{j}^{ \pm}$

$$
\begin{aligned}
Y_{j}^{ \pm}= & -\frac{1}{2} \mathcal{W}^{(j)}(1+\Delta / z)^{-1 / 4} \\
& \left\{\exp \left\{\left[(1+\Delta / z)^{3 / 2}-1\right] \zeta\right\}\left(\sum_{k}(-1)^{k} d_{k} \zeta^{-k}\right)\right. \\
& \left(\sum_{k} c_{k} \zeta^{-k}(1+\Delta / z)^{-3 k / 2}\right) \\
& +\exp \left\{-\left[(1+\Delta / z)^{3 / 2}-1\right] \zeta\right\}\left(\sum_{k} d_{k} \zeta^{-k}\right) \\
& \left.\left(\sum_{k}(-1)^{k} c_{k} \zeta^{-k}(1+\Delta / z)^{-3 k / 2}\right)\right\},
\end{aligned}
$$

for positive arguments, and

$$
\begin{aligned}
Y_{j}^{ \pm}= & -\mathcal{W}^{(j)}(1-\Delta / z)^{-1 / 4} \\
& \left\{\cos \left\{\left[(1-\Delta / z)^{3 / 2}-1\right] \zeta\right\}\right. \\
& {\left[\left(\sum_{k}(-1)^{k} c_{2 k} \zeta^{-2 k}(1-\Delta / z)^{-3 k}\right)\right.} \\
& \left(\sum_{k}(-1)^{k} d_{2 k} \zeta^{-2 k}\right)+\left(\sum_{k}(-1)^{k} d_{2 k+1} \zeta^{-(2 k+1)}\right) \\
& \left.\left(\sum_{k}(-1)^{k} c_{2 k+1} \zeta^{-(2 k+1)}(1-\Delta / z)^{-3(2 k+1) / 2}\right)\right] \\
& \left.+\sin _{2}\left[(1-\Delta / z)^{3 / 2}-1\right] \zeta\right\} \\
& {\left[\left(\sum_{k}(-1)^{k} c_{2 k+1} \zeta^{-(2 k+1)}(1-\Delta / z)^{-3(2 k+1) / 2}\right)\right.} \\
& \left(\sum_{k}(-1)^{k} d_{2 k} \zeta^{-2 k}\right)-\left(\sum_{k}(-1)^{k} d_{2 k+1} \zeta^{-(2 k+1)}\right) \\
& \left.\left.\left(\sum_{k}(-1)^{k} c_{2 k} \zeta^{-2 k}(1-\Delta / z)^{-3 k}\right)\right]\right\},
\end{aligned}
$$

for negative arguments. All the summations are rapidly convergent for $z \gg|\Delta|$. Exponential and trigonometric functions are required for small and accurately determined arguments only, using

$$
\begin{gathered}
(1+x)^{k}=\sum_{i=0}^{\infty}\left(\begin{array}{c}
k \\
i
\end{array}\right) x^{i}, \\
(1+x)^{k}-1=\sum_{i=1}^{\infty}\left(\begin{array}{c}
k \\
i
\end{array}\right) x^{i},
\end{gathered}
$$

for small $x$. Hence, evaluation is free of numerical problems. In numerical evaluation, all the sums are truncated if the value of a term drops below the value of the first term times the machine epsilon.

Finally, we note that our approach is slightly different from that used by Alexander and Manolopoulos, who consider asymptotic expansion of the modulus and phase. ${ }^{8}$ 


\section{APPENDIX B: CONNECTION WITH ENHANCED NUMEROV}

Instead of considering a reference potential with a piecewise definition, one could assume the reference potential constant over each three-point interval, $W^{(i)}(R)=W^{(i)}$. In this case, the independent solutions are the (hyperbolic) trigonometric functions of argument $\sqrt{\left|W^{(i)}\right|} R$. The space of solutions is defined by

$$
\begin{aligned}
A^{(i)} & = \begin{cases}\frac{\sin \left(\sqrt{\left|W^{(i)}\right|}\left(R_{i}-R_{i+1}\right)\right)}{\sin \left(\sqrt{\left|W^{(i)}\right|}\left(R_{i+1}-R_{i-1}\right)\right)} & \text { if } W^{(i)}<0, \\
\frac{\sinh \left(\sqrt{W^{(i)}}\left(R_{i}-R_{i+1}\right)\right)}{\sinh \left(\sqrt{W^{(i)}}\left(R_{i+1}-R_{i-1}\right)\right.} & \text { if } W^{(i)}>0,\end{cases} \\
B^{(i)} & =1, \\
C^{(i)} & = \begin{cases}\frac{\sin \left(\sqrt{\left|W^{(i)}\right|}\left(R_{i-1}-R_{i}\right)\right)}{\sin \left(\sqrt{\left|W^{(i)}\right|}\left(R_{i+1}-R_{i-1}\right)\right)} & \text { if } W^{(i)}<0, \\
\frac{\sinh \left(\sqrt{W^{(i)}}\left(R_{i-1}-R_{i}\right)\right)}{\sinh \left(\sqrt{W^{(i)}}\left(R_{i+1}-R_{i-1}\right)\right)} & \text { if } W^{(i)}>0 .\end{cases}
\end{aligned}
$$

If we choose the grid to be equidistant with spacing $\Delta$, this simplifies to

$$
A^{(i)}=C^{(i)}= \begin{cases}\frac{1}{-2 \cos \left(\sqrt{\left|W^{(i)}\right|} \Delta\right)} & \text { if } W^{(i)}<0, \\ \frac{1}{-2 \cosh \left(\sqrt{W^{(i)}} \Delta\right)} & \text { if } W^{(i)}>0 .\end{cases}
$$

We note that this is equivalent to the enhanced renormalized Numerov method of Thorlacius and Cooper, ${ }^{9}$ a fourth-order solution-following algorithm that becomes exact for constant potentials. Numerically, we found that the order is close to two, if non-equidistant grids are chosen.

${ }^{1}$ B. R. Johnson, J. Chem. Phys. 69, 4678 (1978).

${ }^{2}$ B. R. Johnson, NRCC Proc. 5, 86 (1979).

${ }^{3}$ J. M. Hutson and S. Green, Molscat computer code, version 14, 1994, distributed by Collaborative Computational Project No. 6 of the Engineering and Physical Sciences Research Council (UK).

${ }^{4}$ Hibridon is a package of programs for the time-independent quantum treatment of inelastic collisions and photodissociation written by M. H. Alexan- der, D. Manolopoulos, H.-J. Werner, and B. Follmeg, with contributions by P. Vohralik, G. Corey, B. Johnson, T. Orlikowski, and P. Valiron.

${ }^{5}$ G. W. M. Vissers, G. C. Groenenboom, and A. van der Avoird, J. Chem. Phys. 119, 277 (2003).

${ }^{6}$ G. A. Parker, T. G. Schmalz, and J. C. Light, J. Chem. Phys. 73, 1757 (1980).

${ }^{7}$ L. D. Thomas et al., J. Comput. Phys. 41, 407 (1981)

${ }^{8}$ M. H. Alexander and D. E. Manolopoulos, J. Chem. Phys. 86, 2044 (1987).

${ }^{9}$ A. E. Thorlacius and E. D. Cooper, J. Comput. Phys. 72, 70 (1987).

${ }^{10}$ F. D. Colavecchia, F. Mrugała, G. A. Parker, and R. T Pack, J. Chem. Phys. 118, 10387 (2003)

${ }^{11}$ J. Vigo-Aguiar and H. Ramos, J. Math. Chem. 37, 255 (2005).

${ }^{12}$ D. E. Manolopoulos, J. Chem. Phys. 85, 6425 (1986).

${ }^{13}$ D. E. Manolopoulos and S. K. Gray, J. Chem. Phys. 102, 9214 (1995).

${ }^{14}$ R. G. Gordon, J. Chem. Phys. 51, 14 (1969).

${ }^{15}$ J. C. Light and R. B. Walker, J. Chem. Phys. 65, 4272 (1976).

${ }^{16}$ M. H. Alexander, J. Chem. Phys. 81, 4510 (1984).

${ }^{17}$ M. Kirste, X. Wang, H. C. Schewe, G. Meijer, K. Liu, A. van der Avoird, L. M. C. Janssen, K. B. Gubbels, G. C. Groenenboom, and S. Y. T. van de Meerakker, Science 338, 1060 (2012).

${ }^{18}$ L. M. C. Janssen, P. S. Żuchowski, A. van der Avoird, G. C. Groenenboom, and J. M. Hutson, Phys. Rev. A 83, 022713 (2011).

${ }^{19}$ L. M. C. Janssen, A. van der Avoird, and G. C. Groenenboom, Phys. Rev. Lett. 110, 063201 (2013).

${ }^{20}$ D. W. Chandler, J. Chem. Phys. 132, 110901 (2010).

${ }^{21}$ M. L. González-Martínez and J. M. Hutson, Phys. Rev. A 75, 022702 (2007).

${ }^{22}$ U. Hechtfischer et al., Phys. Rev. Lett. 80, 2809 (1998).

${ }^{23}$ C. J. Williams and K. F. Freed, J. Chem. Phys. 85, 2699 (1986).

${ }^{24}$ K. M. Jones, E. Tiesinga, P. D. Lett, and P. S. Julienne, Rev. Mod. Phys. 78, 483 (2006).

${ }^{25}$ See supplementary material at http://dx.doi.org/10.1063/1.4891809 for SCILAB and MATLAB routines for normalized propagators and test problems using the Numerov, Qsin, and Qairy algorithms.

${ }^{26} \mathrm{~J}$. Stoer and R. Bulirsch, Introduction to Numerical Analysis (SpringerVerlag, New York, 1980).

${ }^{27}$ B. V. Noumerov, Mon. Not. R. Astron. Soc. 84, 592 (1924).

${ }^{28}$ M. Abramowitz and I. A. Stegun, Handbook of Mathematical Functions (National Bureau of Standards, Washington, DC, 1964).

${ }^{29}$ E. J. Rackham, T. Gonzalez-Lezana, and D. E. Manolopoulos, J. Chem. Phys. 119, 12895 (2003)

${ }^{30}$ M. H. Alexander and R. G. Gordon, J. Chem. Phys. 55, 4889 (1971).

${ }^{31}$ D. Secrest and B. R. Johnson, J. Chem. Phys. 45, 4556 (1966).

${ }^{32}$ Scilab: Free and Open Source Software for Numerical Computation (Scilab Enterprises, 2012).

${ }^{33}$ Matlab Version 2013A (The MathWorks, Inc., 2013).

${ }^{34}$ D. E. Amos, "A subroutine package for Bessel functions of a complex argument and nonnegative order," Sandia National Laboratory Report No. 1018, 1985. 